\title{
Análise da taxa de eficácia dos testes sorológicos rápidos para COVID-19 registrados na ANVISA, uma revisão sistemática na literatura
}

Analysis of the effectiveness rate of rapid serological tests for COVID-19 registered in ANVISA, a systematic review in the literature

Análisis de la tasa de efectividad de las pruebas serológicas rápidas para COVID-19 registradas en ANVISA, una revisión sistemática de la literatura

Recebido: 19/08/2021 | Revisado: 25/08/2021 | Aceito: 28/08/2021 | Publicado: 31/08/2021

Lucas Antonio de Oliveira Santos

ORCID: https://orcid.org/0000-0001-6024-0231 Faculdade de Ciências Humanas, Exatas e da Saúde do Piauí, Brasil E-mail: lucasantonio1452@gmail.com

Yuri Dias Macedo Campelo

ORCID: https://orcid.org/0000-0001-7373-3107

Faculdade de Ciências Humanas, Exatas e da Saúde do Piauí, Brasil E-mail: Yuri.campelo@iesvap.edu.br

Renata Paula Lima Beltrão

ORCID: https://orcid.org/0000-0002-3624-6171 Universidade Federal do Piaú, Brasil E-mail:rplbeltrao@gmail.com

Gabriela de Souza Mendonça ORCID: https://orcid.org/0000-0002-7922-5995

Faculdade de Ciências Humanas, Exatas e da Saúde do Piauí, Brasil E-mail:gabyaraujo2007@hotmail.com Viviane Alves da Silva ORCID: https://orcid.org/0000-0002-4973-3219 Faculdade de Ciências Humanas, Exatas e da Saúde do Piauí, Brasil E-mail: vivianealvessilva1996@outlook.com Vanessa Meneses de Brito Campelo ORCID: https://orcid.org/0000-0002-9469-9857 Faculdade de Ciências Humanas, Exatas e da Saúde do Piauí, Brasil E-mail: vanessamenesesbrito@gmail.com

\begin{abstract}
Resumo
Introdução: Os testes imunológicos para a COVID-19 possuem como grande vantagem a sua rapidez diagnóstica, porém possuem taxa de eficácia variável a depender do fabricante, gerando a necessidade de identificar quais os melhores testes a serem utilizados a fim de evitar falsos diagnósticos. Objetivo: Apontar os testes disponíveis no Brasil que, dentro dos ensaios clínicos buscados, apresentam melhor taxa de eficácia. Métodos: Foram buscados na literatura artigos referentes a ensaios clínicos que testam a eficácia dos testes rápidos para a detecção de imunoglobulinas anti-SARS-COV-2. Para tal fim foi realizada uma busca sistemática nas bases de dados SCIELO, LILACS, PUBMED e MEDLINE utilizando os descritores SARS-COV-2, ELISA, COVID-19 e Avaliação, associados utilizando o operador booleano AND, logo em seguida foram aplicados critérios de inclusão e exclusão para selecionar os trabalhos a serem incluídos na revisão. Resultados: Foram incluídos 16 artigos que testaram 18 dos testes rápidos disponíveis no país. Conclusão: Os testes sorológicos são uma ferramenta de grande valia ao enfrentamento da pandemia do SARS-COV-2, porém não devem ser utilizados como ferramenta única no diagnóstico da infecção viral dado o período de janela imunológica. O emprego ideal dos testes dar-se-á nos casos em que o paciente já apresenta mais de 14 dias de sintomas. O teste One Step COVID-2019 da empresa Guangzhou Wondfo Biotech revelou o melhor desempenho dentre os analisados.
\end{abstract}

Palavras-chave: SARS-COV-2; ELISA; COVID-19; Avaliação.

\begin{abstract}
Introduction: The immunological tests for COVID-19 have the great advantage of their diagnostic speed, but their efficiency rate varies depending on the manufacturer, generating the need to identify the best tests to be used in order to avoid false diagnoses. Objective: To point out the tests available in Brazil, which within the clinical trials sought, have a better rate of effectiveness. Methods: Articles referring to clinical trials that test the effectiveness of rapid tests for the detection of anti-SARS-COV-2 immunoglobulins were searched in the literature. For this purpose, a systematic search was carried out in the SCIELO, LILACS, PUBMED and MEDLINE databases using the descriptors
\end{abstract}


SARS-COV-2, ELISA, COVID-19 and Assessment in association using the Boolean operator AND, followed by the application of criteria for inclusion and exclusion to select the papers to be included in the review. Results: 16 articles that tested 18 of the rapid tests available in the country were included. Conclusion: serological tests are a valuable tool to fight the SARS-COV-2 pandemic, but should not be used as a single tool in the diagnosis of viral infection given the period of immunological window. The ideal use of the tests will occur in cases where the patient already has more than 14 days of symptoms. The One Step COVID-2019 test from Guangzhou Wondfo Biotech revealed the best performance among those analyzed.

Keywords: SARS-COV-2; ELISA; COVID-19; Evaluation.

\section{Resumen}

Introducción: Las pruebas inmunológicas para COVID-19 tienen la gran ventaja de su rapidez diagnóstica, pero su tasa de eficiencia varía según el fabricante, generando la necesidad de identificar las mejores pruebas a utilizar para evitar falsos diagnósticos. Objetivo: Señalar las pruebas disponibles en Brasil, que dentro de los ensayos clínicos buscados, tienen una mejor tasa de efectividad. Métodos: Se buscaron en la literatura artículos referentes a ensayos clínicos que prueban la efectividad de las pruebas rápidas para la detección de inmunoglobulinas anti-SARS-COV-2. Para ello, se realizó una búsqueda sistemática en las bases de datos SCIELO, LILACS, PUBMED y MEDLINE utilizando los descriptores SARS-COV-2, ELISA, COVID-19 y Assessment en asociación utilizando el operador booleano AND, seguido de la aplicación de criterios. para su inclusión y exclusión para seleccionar los artículos que se incluirán en la revisión. Resultados: Se incluyeron 16 artículos que probaron 18 de las pruebas rápidas disponibles en el país. Conclusión: las pruebas serológicas son una herramienta valiosa para combatir la pandemia SARS-COV-2, pero no deben utilizarse como una herramienta única en el diagnóstico de infección viral dado el período de ventana inmunológica. El uso ideal de las pruebas se dará en los casos en que el paciente ya tenga más de 14 días de síntomas. La prueba One Step COVID-2019 de Guangzhou Wondfo Biotech reveló el mejor rendimiento entre los analizados.

Palabras clave: SARS-COV-2; ELISA; COVID-19; Evaluación.

\section{Introdução}

Covid-19 (do inglês coronavirus disease 2019) é o termo que denomina o conjunto de manifestações sistêmicas resultantes da infecção pela nova cepa viral da família Coronaviridae, o SARS- CoV- 2. O primeiro registro da doença data de dezembro de 2019, na cidade de Wuhan, na China, período em que se observou um crescente número de pacientes com sintomas respiratórias, digestivos e sistêmicas, que tendiam a ser mais severos em idosos e indivíduos com comorbidades cardiovasculares e metabólicas. Com alta taxa de transmissibilidade, a Covid-19 não demorou a se espalhar além das fronteiras de Wuhan, e galgar o status de pandemia. (Zakary,2020).

O SARS-COV-2 é um vírus de ácido ribonucleico (RNA) de cápsula em formato circular, envolta em proteínas espiculares, proteínas S. As espículas do novo coronavírus possuem afinidade com os receptores da enzima conversora de angiotensina 2 (ECA-2), sendo a ligação com essas estruturas o que possibilita a infecção das células do hospedeiro. Uma vez que os receptores ECA-2 estão dispersos em todo o organismo, o sítio de atuação do SARS-COV-2 é bastante extenso, o que explica a ampla gama de sintomas da COVID-19, que variam em combinação, apresentação e intensidade. (De Souza Carvalho, 2020).

O quadro da COVID-19 também varia de acordo com as características de cada hospedeiro. Além dos órgãos alvo da infecção e carga viral, fatores endógenos como grau de resposta imune, características metabólicas, e comorbidades também influenciam na gravidade dos casos. Pacientes obesos, e ou com distúrbios insulínicos, possuem maior expressão de receptores ECA-2 no tecido adiposo e no pulmão, além de terem risco aumentado para doenças cardiovasculares, nas quais a circulação de citocinas é acrescida. Todos esses fatores propiciam uma maior replicação viral e resposta inflamatória orgânica exacerbada, que somados predispõem a casos graves com maior chance de mortalidade. (Banerjee, 2020).

Os métodos de diagnósticos para a COVID-19, frente ao cenário pandêmico atual, se tornaram uma grande arma para a detecção, controle e isolamento dos casos. Atualmente a detecção da doença pode ser feita através de testes de reação de transcriptase reversa, seguida de reação em cadeia da polimerase (RT-PCR), e da dosagem das imunoglobulinas IgA, IgM e IgG reativas ao vírus, através de imunoensaios de fluxo lateral (LFIAs), ensaios de imunoadsorção enzimática (ELISAs) ou imunoensaios quimioluminescentes (CLIAs). Os exames laboratoriais com base na técnica RT-PCR são testes moleculares que 
identificam a presença do material genético viral em amostras do sangue ou secreções do paciente, são o padrão ouro para a detecção viral, porém possuem o resultado demorado, em torno de dias. Os testes para a detecção das imunoglobulinas identificam a presença de anticorpos para o vírus no organismo, e possuem resultados mais rápidos, em minutos, porém tem taxa de eficácia variante a depender do fabricante e técnica de imunoensaio e do período de infecção do paciente. (Rosón, 2020).

A Agência Nacional de Vigilância Sanitária (ANVISA), vinculada ao Ministério da Saúde, é responsável por aprovar o uso e comercialização dos testes em território nacional. De acordo com o órgão, existem 64 testes para COVID-19 registrados no Brasil, sendo 15 RT-PCR e 49 testes rápidos, sendo 38 testes imunocromatográficos, seis por ELISA, dois imunoensaios por quimioluminescência (CLIA) e três atuam por imunofluorescência (FIA) para a detecção e diferenciação de anticorpos IgA, IgG ou IgM contra o coronavírus (SARS-CoV-2). Nesse contexto, dado o grande número de testes disponíveis, torna se perigoso que não haja muitos estudos que busquem selecionar os testes em circulação por melhor taxa de eficácia, visto os encargos sanitários e sociais que diagnósticos errôneos da COVID-19 podem acarretar no cenário pandêmico. Dados os motivos citados, o presente estudo se propõe a realizar uma revisão sistemática acerca da eficiência dos testes rápidos usados no Brasil para a deteç̧ão da COVID-19, tendo por objetivo apontar os testes, que dentro dos ensaios clínicos buscados, apresentam melhor taxa de eficácia, para que os mesmos possam ser proferidos no diagnóstico da doença. (Ministério da Saúde, 2020; Da Silveira, 2020).

\section{Metodologia}

Critérios de elegibilidade: Artigos originais derivados de ensaios clínicos de estudos de avaliação que apontem a taxa de eficácia de testes rápidos para COVID-19.

Fontes de informação: Artigos originais identificados pela busca em base de dados eletrônicas, SCIELO, LILACS, PUBMED e MEDLINE; a partir de limites para a linguagem e data de publicação especificados no item de seleção dos estudos. Pesquisa: Foram utilizados para busca nos bancos de dados, em associação utilizando o operador booleano AND, os seguintes descritores: SARS-COV-2; ELISA; COVID-19; Avaliação. A busca foi realizada três vezes, utilizando suas correspondentes em cada idioma. Inglês, portugûes e Espanhol. Foram utilizados os filtros de pesquisa: Artigos de avaliação; Texto completo; artigos publicados nos últimos 5 anos. (Clarke, 2001; Cordeiro, 2007).

Seleção dos estudos: inicialmente, foram selecionados artigos de pesquisa com os seguintes critérios de inclusão: Artigos de avaliação derivados de ensaios clínicos de estudos que apontam taxa de eficácia de marcas de testes rápidos para COVID-19. Textos nas línguas português, inglês e espanhol. Após a seleção dos artigos, 2 revisores utilizaram critérios de exclusão para retirar possíveis artigos que não estivessem dentro do escopo da atual revisão. Para isso, os títulos de todos os trabalhos pré-selecionados foram lidos e deveriam fazer referência a testar a eficácia de marcas de testes de imunoglobulinas contra o SARS-COV-2. Em seguida, aplicou-se os mesmos critérios para avaliar a seção resumo dos artigos remanescentes. Processo de coleta de dados: Os dados foram coletados e organizados utilizando uma folha de extração desenvolvida internamente (fundamentada no modelo de extração de dados do Cochrane Consumers and Communication Review Group), testou-a em cinco estudos incluídos aleatoriamente e refinou-a de acordo. Um autor de revisão extraiu os dados de estudos incluídos e o segundo autor verificou os dados extraídos. Os desentendimentos foram resolvidos por discussão entre os dois autores da revisão; se nenhum acordo pudesse ser alcançado, foi planejado que um terceiro autor decidisse. (Clarke, 2001; Cordeiro, 2007).

Compilação, análise e exposição dos dados: Foram destacadas as informações referentes aos testes utilizados em território nacional, presentes na tabela disponibilizada pela ANVISA. As taxas de sensibilidade e especificidade obtidas para cada teste foram reservadas, assim como seus intervalos de confiança (IC). Para os testes que foram avaliados em mais de um 
ensaio clínico, foi adotada a média das taxas obtidas em cada modalidade e calculado um novo intervalo de confiança de $95 \%$ para as mesmas, utilizando o software Bioestat em sua versão 5.3. Após a obtenção dos valores, os testes foram dispostos em tabela em ordem decrescente por critério de taxas de especificidade, sensibilidade em um menor intervalo de confiança associado. A eficácia dos testes foi considerada a partir destes valores. (Clarke, 2001; Cordeiro, 2007; Ministério da Saúde, 2020).

\section{Resultados e Discussão}

Utilizando a associação dos descritores nas bases de dados selecionadas foram obtidos o total de 83 resultados, última pesquisa realizada no dia 03/07/21. Dos resultados obtidos, 36 títulos estavam repetidos, todos os artigos estavam disponíveis na íntegra. Após análise e aplicação dos critérios de inclusão e exclusão por nossos revisores, foram incluídos 16 estudos nesta revisão.

Os testes rápidos para detecção da COVID-19 nas várias modalidades técnicas disponíveis no mercado, possuem o viés da resposta imunológica, uma vez que o diagnóstico da patologia só se torna possível alguns dias após o início da sintomatologia. Cota através de seu estudo utilizando amostras sorológicas de 147 pacientes RT-PCR positivos, em períodos distintos de sintomatologia, os testes sorológicos para a covid analisados revelaram baixo valor para o diagnóstico individual nos primeiros 7 dias após o início dos sintomas. Corroboraram para esta mesma conclusão os resultados obtidos nos experimentos realizados por Nilsson, Gutiérrez-Cobos e Chansaenroj. (Cota, 2020; Nilsson, 2021; Gutiérrez-Cobos, 2021; Chansaenroj, 2021).

Ainda sobre a janela imunológica, dos estudos incluídos que utilizaram amostras de pacientes em períodos variados de sintomatologia, observou-se uma alta geral significativa na sensibilidade dos testes sorológicos, para amostras obtidas de pacientes com mais de 14 dias de sintomas, considerando este como tempo médio para soroconversão. Outrossim, outros ensaios convergem de maneira semelhante, a exemplo Whitman constatou alta na sensibilidade dos testes após 20 dias de sintomatologia, e Van Elslande relata ainda que atingiu 100\% de sensibilidade para os testes incluídos em sua análise, frente ao soro de pacientes com mais de 3 semanas do início dos sintomas. (Whitman, D,2020; Van Elslande, 2020).

Dos autores incluídos nesta revisão 14 dos 16, concluíram que os testes sorológicos são eficazes e úteis para o diagnóstico da COVID-19 dentro da janela de expressão orgânica das imunoglobulinas. Whitman afirma que os testes são ferramentas essenciais na resposta à pandemia. Montesinos reconhece e aponta os imunoensaios como ferramentas para a retomada gradual da atividade econômica e social com segurança. Beavis ressalta que os testes imunológicos não devem ser utilizados como fonte exclusiva para o diagnóstico, evidenciando a necessidade da clínica e dos exames complementares. Os outros dois autores não deixam clara suas opiniões, focando-se apenas na análise do desempenho e acurácia dos testes. (Whitman, 2020; Beavis, 2020; Montesinos, 2020).

Costa e Tuaillon concordam que os testes sorológicos podem ser usados para o diagnóstico de pacientes RT-PCR negativos quando clínica e exames de imagem forem sugestivos. O estudo conduzido por Costa mostrou que o imunoensaio cromatográfico analisado foi capaz de positivar o diagnóstico de COVID-19 em 15 dos 16 pacientes com diagnóstico clínico baseado em sintomas altamente sugestivos e achados em tomografia computadorizada (TC) de tórax, embora RT-PCR negativos. Ademais, a análise desenvolvida por Chansaenroj constatou a aplicabilidade do uso de testes de imunoglobulinas anti SARS-COV-2 para diagnóstico de pacientes assintomáticos. (Tuaillon, E, 2020; Costa, S. F, 2020; Chansaenroj, J, 2021).

Após a seleção dos estudos foi aplicada a folha de extração de dados a fim de separar as informações referentes aos testes aprovados pela ANVISA, tendo por base as informações dispostas pelo ministério da saúde. Durante o levantamento de dados desta revisão, foram encontrados ensaios clínicos que testaram a eficácia de 18 dos testes sorológicos aprovados pela ANVISA. (Ministério da Saúde, 2020). 
A sensibilidade e a especificidade foram fatores alvo das verificações desenvolvidas pelos autores. Entende-se por sensibilidade: proporção de testes positivos entre os indivíduos doentes; e especificidade: proporção de testes negativos entre indivíduos não doentes. (Cota, 2020).

Dentre as metodologias adotadas para a aferição da sensibilidade dos ensaios estudados a mais comum foi a porcentagem de soropositivos conseguida utilizando o sangue de pacientes RT-PCR positivos para COVID-19, e dentre as metodologias adotadas para calcular a especificidade dos testes as mais comuns foram a utilização de amostras sorológicas anteriores ao período da pandemia e ou exposição dos testes a amostras contendo anticorpos de adenovírus semelhantes ao SARS-COV-2. (Cramer, 2021).

Anti-SARS-CoV-2 IgG ELISA fabricado pela empresa Alemã Euroimmun, ganhou demasiado destaque, uma vez que foi testado em sensibilidade e ou especificidade por 14 dos ensaios incluídos. Dentro destes estudos a taxa de sensibilidade referida variou bastante, com valores entre $37,8 \%$ e $93,3 \%$, a sensibilidade média obtida entre os estudos foi de 74,46\% (66,8982,04 IC 95\%). Havendo uma observação comum entre os ensaios, que utilizaram soros em tempos de evolução distintos, apontando crescimento exponencial das taxas de sensibilidade obtidas inicialmente, quando analisado o desempenho do teste com amostras de pacientes com mais de 14 dias de sintomatologia, resultando em uma sensibilidade média de 87,01\% (79,8194,21 IC 95\%). Gutiérrez-Cobos, que apontou a menor taxa de sensibilidade ao teste sorológico, 37,8\%, reconhece que foge da curva e levanta limitações que podem justificar o valor discrepante. Dentre as limitações foram citadas amostra reduzida, congelamento e descongelamento excessivos das amostras, ademais, a metodologia do estudo também foi apontada como fator limitante. No que tange a especificidade, os valores não se mostram tão discrepantes, apresentando valores entre $89,2 \%$ e 100\%, e média de 97,23\% (95,38-99,08 IC 95\%). (Gutiérrez-Cobos, 2021; Kohmer, 2020).

O Anti-SARS-CoV-2 IgG ELISA IgA, também fabricado pela empresa alemã Euroimmum, é o segundo colocado com relação ao número de análises, sendo testado por 9 imunoensaios diferentes, apresentando sensibilidade variando entre $82,9 \%$ e $93,1 \%$, sensibilidade média apurada de $86,38 \%$ (82,71-90,05 IC 95\%) e especificidade entre $73 \%$ e $88,4 \%$, com média de 80,58\% (76,55-84,61 IC 95\%). Os baixos níveis de especificidade do teste levaram Nilsson a não recomendar seu uso na triagem inicial dos pacientes dada a alta quantidade de falsos positivos, no estudo em questão a taxa de especificidade obtida para o teste foi de $73,2 \%$. Em geral observou-se uma taxa de sensibilidade mais elevada do teste IgA nos primeiros dias de sintomatologia comparado ao teste IgG do mesmo laboratório. (Nilsson, 2020).

$\mathrm{O}$ terceiro colocado no quesito quantidade de testagens foi o teste $\mathrm{IgM} / \mathrm{IgG}$ da empresa Guangzhou Wondfo Biotech Co.,Ltda, de nome comercial One Step COVID-19. Sendo avaliado em 4 dos ensaios incluídos, apresentando sensibilidade referida entre 94,9\% e 79,7\%, com média de 89,65\% (83,72-95,57 IC 95\%) e especificidade entre $100 \%$ e 95,2\%, média de 98,57\% (96,63-100 IC 95\%). Na quarta posição dentre os mais testados estão os testes Innovita 2019 n-CoV Ab Test Colloidal Gold IgM e IgG, tendo sido avaliados por dois estudos. O teste IgG apresentou sensibilidade média de 61,05\% (37,42-84,67 IC 95\%) e especificidade média de 99\% (97,61-100 IC 95\%). O teste IgM acusou sensibilidades e especificidades médias de respectivamente 45\% (36,1-54,5 IC 95\%) e 98,15\% (95-100 IC 95\%). (Whitman, 2020; Gutiérrez-Cobos, 2021).

O teste Diagnostic Kit for IgM/IgG Antibody to Coronavirus da empresa Zhuhai Livzon Diagnositic Inc. foi avaliado juntamente com o teste Família Teste Rápido em Cassete 2019-nCoV IgG/IgM da empresa fabricante ACRO Biotech Inc, no ensaio clínico promovido por Tuaillon, no qual foram utilizadas amostras de plasma coletadas de pacientes hospitalizados no Hospital Universitário de Montpellier com COVID-19 comprovado ou suspeito por PCR. Os dados obtidos de sensibilidade e especificidade estão dispostos em comparativo com os demais na tabela 1. (Tuaillon, 2020).

Os demais nove testes restantes foram avaliados somente por um mesmo ensaio, o estudo conduzido por Cota, no Brasil, teve por objetivo avaliar randomicamente 12 sorológicos disponíveis para a deteç̧ão da COVID-19 no país. O ensaio clínico em questão utilizou 289 amostras de soro de 173 pacientes sintomáticos com infecção confirmada por SARS-CoV-2 
por testes RT-PCR e 116 controles negativos. Os valores de sensibilidade e especificidade obtidos no estudo, assim como a identificação destes imunoensaios estão dispostos na Tabela 1 em comparação com os outros ensaios citados anteriormente. (Cota,G, 2020).

Tabela 1: Valores de sensibilidade e especificidade do estudo.

\begin{tabular}{|c|c|c|}
\hline Teste (empresa) & $\begin{array}{c}\text { Sensibilidade\% (Intervalo de } \\
\text { confiança 95\%) }\end{array}$ & $\begin{array}{l}\text { Especificidade (Intervalo de } \\
\text { confiança 95\%) }\end{array}$ \\
\hline $\begin{array}{c}\text { Teste One Step COVID-2019 (Guangzhou Wondfo } \\
\text { Biotech) }\end{array}$ & $86,65(83,72-95,57)$ & $98,57(96,63-100)$ \\
\hline COVID-19 ECO IGM / IGG (Eco Diagnostica) & $83,1(76,2-88,3)$ & $99,1(95,2-99,8)$ \\
\hline $\begin{array}{l}\text { Imuno-Rápido COVID-19 IgG / IgM (Wama } \\
\text { Produtos Para Laboratorio) }\end{array}$ & $75,0(67,5-81,3)$ & $97,4(92,6-99,1)$ \\
\hline Anti-SARS-CoV-2 ELISA IgG (Euroimmun) & $74,4(66,89-82,04)$ & $97,23(95,38-99,0)$ \\
\hline Allserum EIA COVID-19 IgG (Mbiolog) & $66,9(58,8-74,1)$ & $98,1(93,4-99,5)$ \\
\hline COVID-19 IgG IgM (Gold Analisa Diagnóstica) & $64,9(56,9-72,1)$ & $98,3(93,9-99,6)$ \\
\hline COVID-19 IgG / IgM (Qingdao Hightop Biotech) & $59,5(51,4-67,1)$ & $100(96,8-100)$ \\
\hline $\begin{array}{c}\text { INNOVITA COVID-19 } \\
\text { IgM (Innovita Biological Technology Co., Ltd.) }\end{array}$ & $45(36,1-54,5)$ & $98,15(95-100)$ \\
\hline $\begin{array}{l}\text { TR DPP® COVID-19 IGM / IGG Bio- } \\
\text { Manguinhos (Fundação Oswaldo Cruz) }\end{array}$ & $73,6(65,9-80,0)$ & $81,0(72,9-87,1)$ \\
\hline Anti-SARS-CoV-2 ELISA IgA (Euroimmun) & $86,38(82,71-90,05)$ & $80,56(76,55-84,61)$ \\
\hline Allserum EIA COVID-19 IgM (Mbiolog) & $50,7(42,6-58,8)$ & $70,4(61,2-78,2)$ \\
\hline Allserum EIA COVID-19 IgM (Mbiolog) & $83,8(82,7-92,9)$ & $53,4(47,4-59,3)$ \\
\hline $\begin{array}{l}\text { COVID-19 ELISA IgA/IgM (Vircell } \\
\text { Microbiologists) }\end{array}$ & $92.6(87.2-95.8)$ & $23.3(16.5-31.8)$ \\
\hline $\begin{array}{l}\text { 2019-nCoV } \operatorname{IgM} / \operatorname{IgG} \text { Antibody Test Kit (Zhuhai } \\
\text { Livzon) *IgG }\end{array}$ & $86,7(69,5-100)$ & 100 \\
\hline $\begin{array}{c}\text { Família Teste Rápido em Cassete 2019-nCoV } \\
\text { IgG/IgM (ACRO Biotech Inc ). IgG* }\end{array}$ & $84,6(65-100)$ & 100 \\
\hline $\begin{array}{l}\text { 2019-nCoV IgM/IgG Antibody Test Kit (Zhuhai } \\
\text { Livzon) *IgM }\end{array}$ & $80.0(59.8-100)$ & $95.0(85.4-100)$ \\
\hline $\begin{array}{c}\text { Família Teste Rápido em Cassete 2019-nCoV } \\
\text { IgG/IgM ( ACRO Biotech Inc ). IgM* }\end{array}$ & $76.9(54.0-99.8)$ & 100 \\
\hline $\begin{array}{c}\text { INNOVITA COVID-19 } \\
\text { IgG (Innovita Biological Technology Co., Ltd.) }\end{array}$ & $61,05(37,42-84,67)$ & $99(97,61-100)$ \\
\hline
\end{tabular}

Nota: Tabela comparativa entre os testes sorológicos avaliados nos artigos incluídos; utilizada ordem dada primeiramente por critério de maior especificidade média, e em segundo lugar levando em consideração a sensibilidade média. (“*” Aplicado para especificar a qual imunoglobulina as taxas do teste se referem). Fonte: Autores. 


\section{Conclusão}

Após análise dos dados obtidos nos ensaios clínicos incluídos nesta revisão, conclui-se que os testes sorológicos são uma ferramenta de grande valia ao enfrentamento da pandemia do SARS-COV-2, porém não devem ser utilizados como ferramenta única no diagnóstico da infecção viral dado o período de janela imunológica. Desse modo, um resultado negativo não exclui a possibilidade de infecção viral, principalmente nos primeiros 7 dias de sintomatologia. O emprego ideal dos testes dar-se-á nos casos em que o paciente já apresenta mais de 14 dias de sintomas. O resultado positivo do teste sorológico aliado a clínica e achados em exames de imagem sugestivos pode ser utilizado para fechar diagnóstico mesmo em casos RT-PCR negativos. O teste One Step COVID-2019 da empresa Guangzhou Wondfo Biotech revelou o melhor desempenho dentre os analisados.

A equipe do artigo atual recomenda aos autores de trabalhos futuros que ao decidirem por adotar metodologia semelhante a esta, deem predileção a incluir a maior quantidade de dados disponíveis, de ensaios clínicos, acerca de um determinado teste, para que se possa afirmar suas características em um menor intervalo de confiança possível e assim, minimizar possíveis vieses.

\section{Referências}

Banerjee, M., Gupta, S., Sharma, P., Shekhawat, J., \& Gauba, K. (2020). Obesity and COVID-19: a fatal alliance. Indian Journal of Clinical Biochemistry, 18 .

Beavis, K. G., Matushek, S. M., Abeleda, A. P. F., Bethel, C., Hunt, C., Gillen, S., \& Tesic, V. (2020). Evaluation of the EUROIMMUN Anti-SARS-CoV-2 ELISA Assay for detection of IgA and IgG antibodies. Journal of Clinical Virology, 129, 104468.

Chansaenroj, J., Yorsaeng, R., Posuwan, N., Puenpa, J., Sudhinaraset, N., Chirathaworn, C., \& Poovorawan, Y. (2021). Detection of SARS-CoV-2-specific antibodies via rapid diagnostic immunoassays in COVID-19 patients. Virology Journal, 18(1), 1-7.

Clarke, M., \& Horton, R. (2001). Reunindo tudo: Lancet-Cochrane colaboram em revisões sistemáticas. The Lancet, 357 (9270), 1728.

Cordeiro, A. M., Oliveira, G. M. D., Rentería, J. M., \& Guimarães, C. A. (2007). Revisão sistemática: uma revisão narrativa. Revista do Colégio Brasileiro de Cirurgiões, 34, 428-431.

Costa, S. F., Buss, L., Espinoza, E. P. S., Vieira Jr, J. M., da Silva, L. C. D. O., de Souza, R. M., \& Sabino, E. (2020). Performance of a qualitative rapid chromatographic immunoassay to diagnose COVID-19 in patients in a middle-income country. Journal of Clinical Virology, $131,104592$.

Cota, G., Freire, M. L., de Souza, C. S., Pedras, M. J., Saliba, J. W., Faria, V., \& Avelar, D. M. (2020). Diagnostic performance of commercially available COVID-19 serology tests in Brazil. International Journal of Infectious Diseases, 101, 382-390.

Cramer, A., Goodman, N., Cross, T., Gant, V., \& Dziadzio, M. (2021). Analytical evaluation and critical appraisal of early commercial SARS-CoV-2 immunoassays for routine use in a diagnostic laboratory. British Journal of Biomedical Science, 78(3), 141-146.

Da Silveira, M. C., \& de Azeredo Costa, E. (2020). Busca ativa ou testagem em massa? Cadernos Ibero-Americanos De Direito Sanitário, 9(4), 188-191.

de Souza Carvalho, F. R., Gobbi, L. C., Carrijo-Carvalho, L. C., Caetano, A. J. F., Casotti, G. C., Tiussi, L. M., \& Cavalari, A. L. C. (2020). FISIOPATOLOGIA DA COVID-19: REPERCUSSÕES SISTÊMICAS. Unesc em Revista, 4(2), 170-184.

Gutiérrez-Cobos, A., de Frutos, S. G., García, D. D., Lara, E. N., Carrión, A. Y., García-Rodrigo, L. F., \& Domingo, L. C. (2021). Evaluation of diagnostic accuracy of 10 serological assays for detection of SARS-CoV-2 antibodies. European Journal of Clinical Microbiology \& Infectious Diseases, 40(5), 955-961.

Kohmer, N., Westhaus, S., Rühl, C., Ciesek, S., \& Rabenau, H. F. (2020). Brief clinical evaluation of six high-throughput SARS-CoV-2 IgG antibody assays. Journal of Clinical Virology, 129, 104480.

Ministério da Saúde (BR). (2020). Coronavírus Covid-19-Acurácia dos testes diagnósticos registrados na ANVISA para a COVID-19.

Montesinos, I., Gruson, D., Kabamba, B., Dahma, H., Van den Wijngaert, S., Reza, S., \& Rodriguez-Villalobos, H. (2020). Evaluation of two automated and three rapid lateral flow immunoassays for the detection of anti-SARS-CoV-2 antibodies. Journal of Clinical Virology, $128,104413$.

Nilsson, A. C., Holm, D. K., Justesen, U. S., Gorm-Jensen, T., Andersen, N. S., Øvrehus, A., \& Lillevang, S. T. (2021). Comparison of six commercially available SARS-CoV-2 antibody assays - Choice of assay depends on intended use. International Journal of Infectious Diseases, 103, $381-388$.

Rosón, P., Pisula, P., Báez, G., Loza, C., Taito, I., Cisneros, V., \& Franco, J. V. A. (2020). Métodos diagnósticos para la infección por SARS-CoV-2. Rev. Hosp. Ital. B. Aires (2004), 117-125.

Tuaillon, E., Bollore, K., Pisoni, A., Debiesse, S., Renault, C., Marie, S., \& Van de Perre, P. (2020). Detection of SARS-CoV-2 antibodies using commercial assays and seroconversion patterns in hospitalized patients. Journal of Infection, 81(2), e39-e45. 
Research, Society and Development, v. 10, n. 11, e264101119615, 2021

(CC BY 4.0) | ISSN 2525-3409 | DOI: http://dx.doi.org/10.33448/rsd-v10i11.19615

Van Elslande, J., Decru, B., Jonckheere, S., Van Wijngaerden, E., Houben, E., Vandecandelaere, P., \& Vermeersch, P. (2020). Antibody response against SARS-CoV-2 spike protein and nucleoprotein evaluated by four automated immunoassays and three ELISAs. Clinical Microbiology and Infection, 26(11), 1557-e1.

Whitman, J. D., Hiatt, J., Mowery, C. T., Shy, B. R., Yu, R., Yamamoto, T. N., \& Marson, A. (2020). Evaluation of SARS-CoV-2 serology assays reveals a range of test performance. Nature biotechnology, 38(10), 1174-1183.

Zakary, O., Bidah, S., \& Rachik, M. (2020). The Impact of Staying Home on Controlling the Propagation of COVID -19: Control Strategy. Mexican Journal of Biomedical Engineering, 42 (1), 10-26. 